\section{Tissue Cells Feel and Respond to the Stiffness of Their Substrate}

\author{
Dennis E. Discher, ${ }^{1 *}$ Paul Janmey, ${ }^{1}$ Yu-li Wang ${ }^{2}$
}

\begin{abstract}
Normal tissue cells are generally not viable when suspended in a fluid and are therefore said to be anchorage dependent. Such cells must adhere to a solid, but a solid can be as rigid as glass or softer than a baby's skin. The behavior of some cells on soft materials is characteristic of important phenotypes; for example, cell growth on soft agar gels is used to identify cancer cells. However, an understanding of how tissue cells-including fibroblasts, myocytes, neurons, and other cell types-sense matrix stiffness is just emerging with quantitative studies of cells adhering to gels (or to other cells) with which elasticity can be tuned to approximate that of tissues. Key roles in molecular pathways are played by adhesion complexes and the actinmyosin cytoskeleton, whose contractile forces are transmitted through transcellular structures. The feedback of local matrix stiffness on cell state likely has important implications for development, differentiation, disease, and regeneration.
\end{abstract}

Anchorage dependence refers to a cell's need for adhesion to a solid. Most tissue cells are simply not viable upon dissociation and suspension in a fluid, even if soluble proteins are added to engage cell adhesion molecules [e.g., integrin-binding RGD peptide $(1,2)]$. Fluids are clearly distinct from solids in that fluids will flow when stressed, whereas solids have the ability to resist sustained pushing and pulling. In most soft tissues - skin, muscle, brain, etc. - adherent cells plus extracellular matrix contribute together to establish a relatively elastic microenvironment. At the macro scale, elasticity is evident in a solid tissue's ability to recover its shape within seconds after mild poking and pinching, or even after sustained compression, such as sitting.

At the cellular scale, normal tissue cells probe elasticity as they anchor and pull on their surroundings. Such processes are dependent in part on myosin-based contractility and transcellular adhesions - centered on integrins, cadherins, and perhaps other adhesion molecules - to transmit forces to substrates. A normal tissue cell not only applies forces but also, as reviewed here, responds through cytoskeleton organization (and other cellular processes) to the resistance that the cell senses, regardless of whether the resistance derives from normal tissue matrix, synthetic substrate, or even an adjacent cell. Furthermore, physical properties of tissues can change in disease [as imaged now by magnetic resonance imaging (MRI) or ultrasound elastography (3-5)], and cellular responsiveness to matrix solidity can

\footnotetext{
${ }^{1}$ School of Engineering and Applied Science and Cell and Molecular Biology Graduate Group, University of Pennsylvania, Philadelphia, PA 19104-6315, USA. ${ }^{2}$ Departments of Physiology and Cell Biology, University of Massachusetts, Worcester, MA 01655, USA.

*To whom correspondence should be addressed. E-mail: discher@seas.upenn.edu
}

likewise change, as illustrated by the growth of cancer cells on soft agar [e.g., (6)].

Contractile forces in cells are generated by cross-bridging interactions of actin and myosin filaments. For adherent cells, some of these forces are transmitted to the substrate (referred to as traction forces) and cause wrinkles or strains when the substrate consists of a thin film or a soft gel (7-12) (Fig. 1A). The cell, in

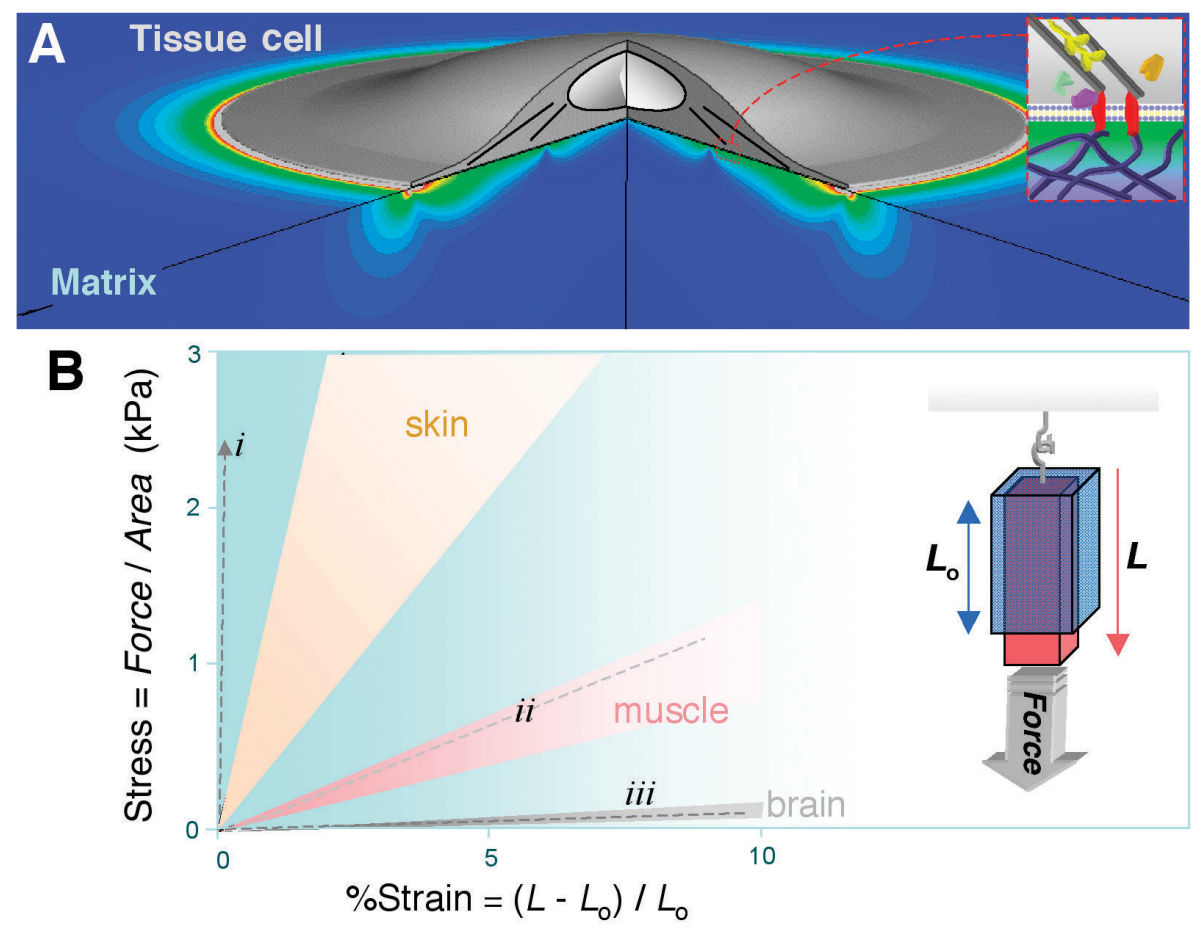

Fig. 1. Substrate strain and tissue stiffness. (A) Strain distribution computed in a soft matrix beneath a cell. The circular cell has a uniform and sustained contractile prestress from the edge to near the nucleus (81). (B) Stress versus strain illustrated for several soft tissues extended by a force (per cross-sectional area). The range of slopes for these soft tissues subjected to a small strain gives the range of Young's elastic modulus, $E$, for each tissue $(24,28,30)$. Measurements are typically made on time scales of seconds to minutes and are in SI units of Pascal (Pa). The dashed lines (---) are those for (i) PLA, a common tissue-engineering polymer (89); (ii) artery-derived acellularized matrix (90); and (iii) matrigel (42). 
tinctly to soft versus stiff substrates. Although molecular pathways are still only partially known, muscle cells, neurons, and many other tissue cells have since been shown to sense substrate stiffness (15-17). Unlike cells on soft gels or in tissues, cells cultured on tissueculture plastic or glass coverslips are attached (often via adsorbed matrix protein) to essentially rigid materials. The question therefore arises: Do cells perceive and respond to the rigidity of these conventional materials in ways that contrast with their behavior in much more compliant tissues, gels, or sublayers of cells? The increasingly clear, affirmative answer to this question appears important in its impact not just on standard cell culture but also, perhaps, in understanding disease processes, morphogenesis, and tissue-repair strategies.

\section{Soft Tissue Benchmarks}

Cells adhere to solid substrates that range in stiffness from soft to rigid and that also vary in topography and thickness (e.g., basement membrane). Regardless of geometry, the intrinsic resistance of a solid to a stress is measured by the solid's elastic modulus $E$, which is most simply obtained by applying a force-such as hanging a weight - to a section of tissue or other material and then measuring the relative change in length or strain (Fig. 1B, inset). Another common method to obtain $E$ involves controlled poking by macro- and micro-indenters, including atomic force microscopes (AFMs) $(18,19)$. Many tissues and biomaterials exhibit a relatively linear stress versus strain relation up to small strains of about 10 to $20 \%$. The slope $E$ of stress versus strain is relatively constant at the small strains exerted by cells (20), although stiffening (increased $E$ ) at higher strains is the norm $(21,22)$. Nonetheless, microscopic views of both natural and synthetic matrices [e.g., collagen fibrils and polymer-based mimetics (23)] suggest that there are many subtleties to tissue mechanics, particularly concerning the length and time scales of greatest relevance to cell sensing. Sample preparation or state is another obvious issue; for example, elastic moduli of whole brain in macroscopic measurements can vary by a factor of 2 or more, depending on specifics of preparation, tissue perfusion, etc. (24). In addition, with cells as well as tissues, many probing methods involve high-frequency stressing (25), whereas relevant time scales for cell-exerted strains seem likely to range from seconds to hours, motivating long time studies of cell rheology [recent cell mechanics references $(26,27)]$. Regardless, comparisons of three diverse tissues that contain a number of different cell types show that brain tissue is softer than muscle $(28,29)$, and muscle is softer than skin (30) (Fig. 1B). Although mapping soft tissue micro-elasticities at a resolution typical in histology seems important, the implication here is that there are dis- tinct elastic microenvironments for epithelial cells and fibroblasts in skin, for myotubes in fiber bundles, and for neurons in brain.

Correlations have long been made between increased cell adhesion and increased cell contractility [e.g., (31)], but it now seems clear that tactile sensing of substrate stiffness feeds back on adhesion and cytoskeleton, as well as on net contractile forces, for many cell types. Seminal studies on epithelial cells and fibroblasts exploited inert polyacrylamide gels with a thin coating of covalently attached collagen (14). This adhesive ligand allows the cells to attach and-by controlling the extent of polymer cross-linking in the gels $-E$ can be adjusted over several orders of magnitude, from extremely soft to stiff. Images of adhesion proteins such as vinculin are revealing (Fig. 2, top): Soft, lightly cross-linked gels $(E \sim 1 \mathrm{kPa})$ show diffuse and dynamic adhesion complexes. In contrast, stiff, highly crosslinked gels $(E \sim 30$ to $100 \mathrm{kPa})$ show cells with stable focal adhesions, typical of those seen in cells attached to glass. Similarly, rigidification of cell-derived three-dimensional (3D) matrices alters 3D-matrix adhesions, because the adhesions are replaced by large, nonfibrillar focal adhesions similar to those found on fixed 2D substrates of fibronectin (32). Consistent with a role for signaling in stiffness sensing, tyrosine phosphorylation on multiple proteins (including paxillin) appears broadly enhanced in cells on stiffer gel substrates (14), whereas pharmacologically induced, nonspecific hyperphosphorylation drives focal adhesion formation on soft materials. Inhibition of actomyosin contractions, in contrast, largely eliminates prominent focal adhesions, whereas stimulation of contractility drives integrin aggregation into adhesions (33). Additionally, although microtubules have been proposed to act as "struts" in cells and thus limit wrinkling of thin films by cells (34), quantification of their contributions to cells on gels shows that they provide only a minor fraction of the resistance $(14 \%)$ to contractile tensions; most of a cell's tension is thus resisted by matrix (35).

Traction stresses ( $\tau$, force per area) exerted by fibroblasts on gels were the first to be mapped by embedding fluorescent microbeads near the gel surface and then imaging bead displacements before and after cell detachment $(10,20)$. Although larger tractions are exerted on stiffer gels, typical tractions of $\langle\tau\rangle \sim 1 \mathrm{kPa}$ exceed by orders of magnitude the viscous fluid drag on any cell crawling in culture. In addition, mean cell tractions equate to mean gel strains that differ very little $\left(\varepsilon_{\text {out }}=\langle\tau / E\rangle \cong\right.$ 3 to $4 \%$ ) between gels that differ by twofold in $E$. This suggests that $\varepsilon_{\text {out }}$ is sensed by cells as a tactile set-point, perhaps analogous to other physiological set-points such as extracellular ion concentrations or optimal growth factor concentrations. Furthermore, if matrix strain is approximately constant, then cells on soft gels need be less contractile than on stiff gels, and if they are less contractile, then
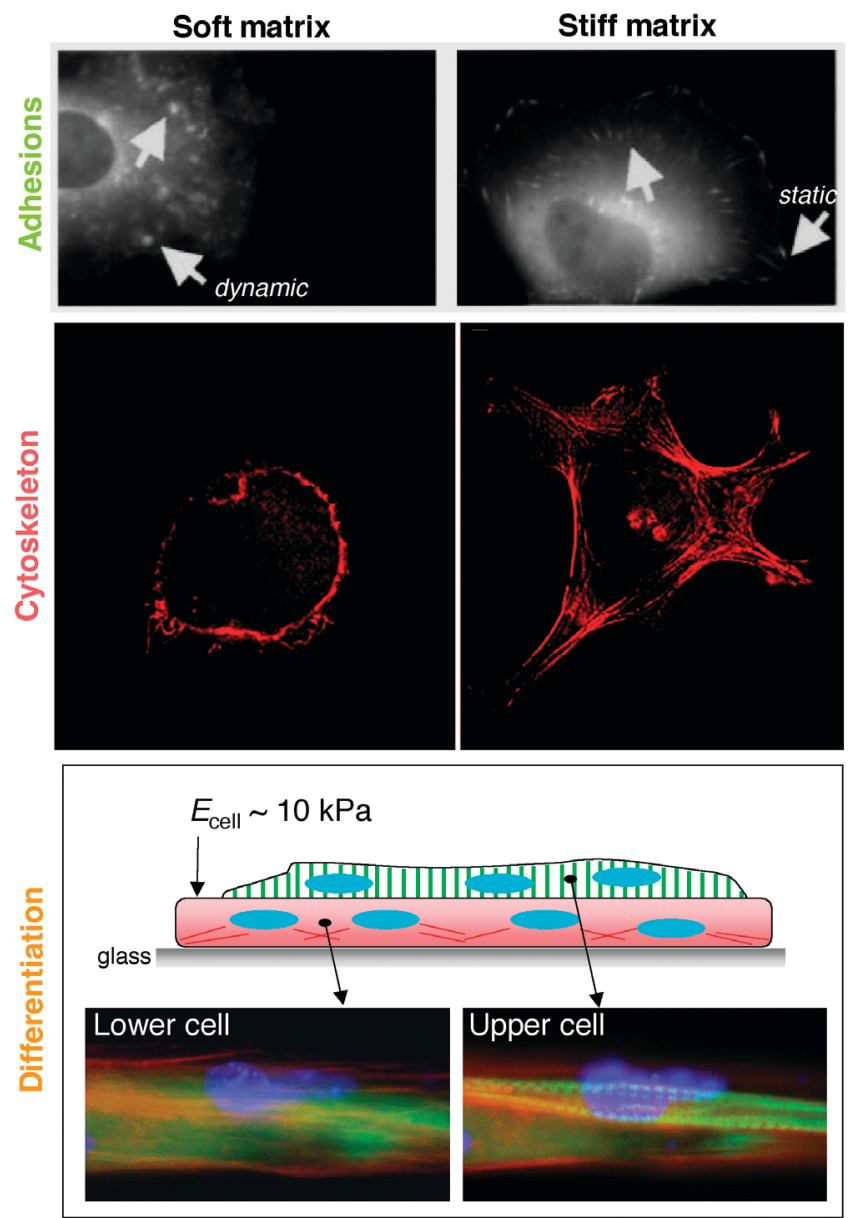

Fig. 2. Substrate stiffness influences adhesion structures and dynamics (14), cytoskeleton assembly and cell spreading (17, 42), and differentiation processes such as striation of myotubes (28). (Top) The arrows point to dynamic adhesions on soft gels and static, focal adhesions on stiff gels. [Adapted from (14)] (Middle) The actin cytoskeleton. (Bottom) A cell-on-cell layering in which the lower layer is attached first to glass so that the upper layer, which fuses from myoblasts that are added later, perceives a soft, cellular substrate. 
their adhesions need not be as strong. This is consistent with a reduced adhesion strength as measured by reduced forces to peel cells from soft gels versus glass (28). This is also consistent with more dynamic adhesions on soft substrates (Fig. 2, top). Fluorescence imaging also shows increasingly organized F-actin and stress fibers on increasingly stiff substrates in fibroblasts (Fig. 2, middle). Neurons, in contrast, appear to apply very little stress to their substrate, because they can only deform very soft gels (36). Neurons also branch more on softer substrates (37), perhaps because the cytoskeleton is more pliable, if less structured.

\section{Differentiation and a \\ Cell-on-Cell Hypothesis}

Cytoskeletal organization in muscle cells also depends on substrate stiffness and reveals an optimal substrate stiffness for striation of actomyosin $(28,38)$ - the contractile element of the myotube. On very soft gels that are micropatterned with collagen strips so as to generate well-separated myotubes, actomyosin appears diffuse after weeks in culture. On very stiff gels, as well as on glass micropatterns, stress fibers and strong focal adhesions predominate, suggesting a state of isometric contraction. Notably, however, on gels with an
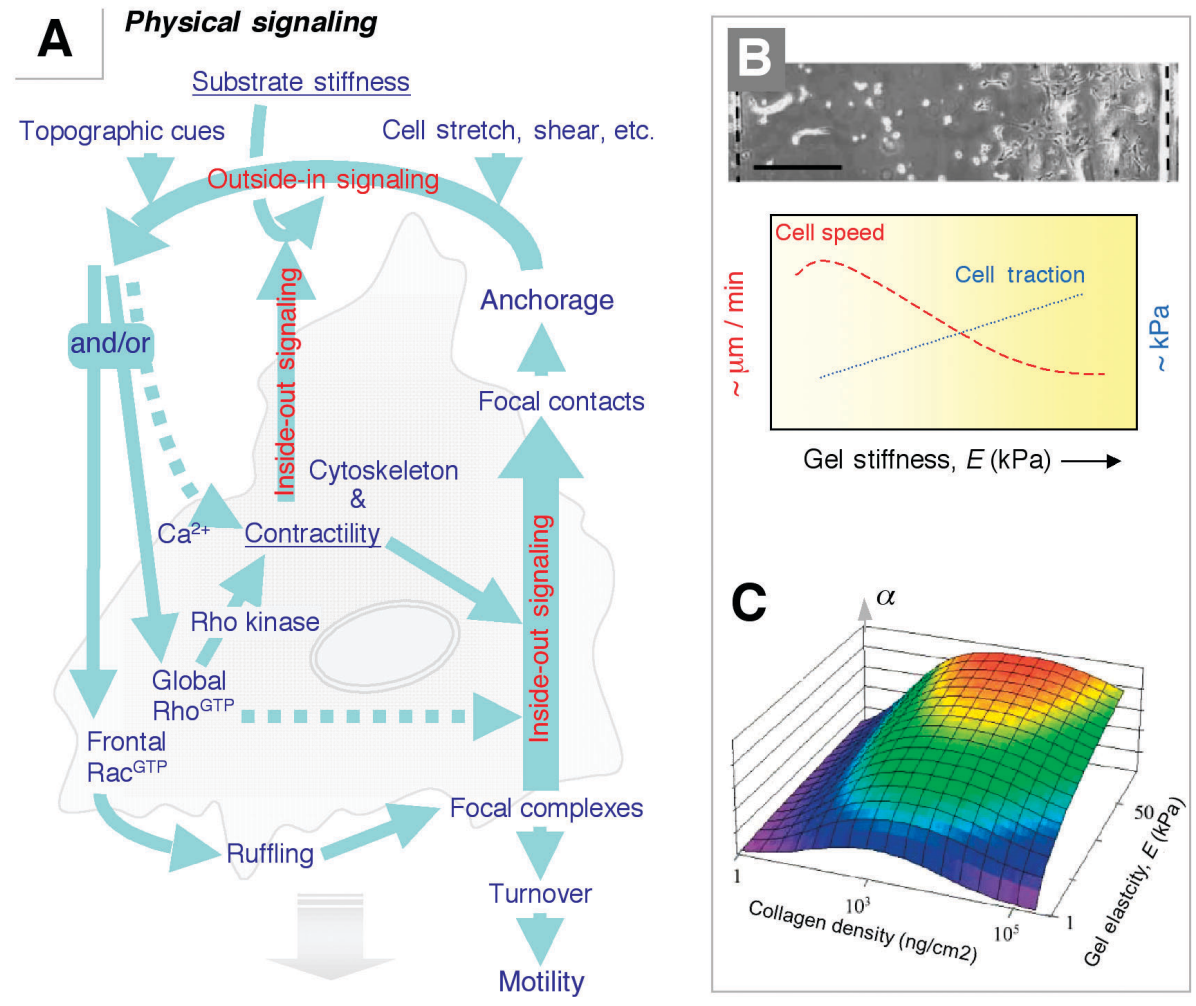

Fig. 3. Substrate stiffness influences contractility, motility, and spreading. (A) Interplay of physical and biochemical signals in the feedback of matrix stiffness on contractility and cell signaling as extended from (91). (B) Cells exert less tension on softer, collagen-coated gels but crawl faster (20), causing an accumulation of cells toward the stiff end of a soft-to-stiff gradient gel (54). Curves are schematic. [Image adapted from (54)] (C) Spread area, $\alpha$, of smooth muscle cell versus ligand density and matrix stiffness, based on measurements fitted by a thermodynamic model (17). Similar nonlinear responses are also seen for adhesions, cytoskeleton organization, tractions exerted on the substrate, and other cellular processes. with epithelial cells and fibroblasts, as well as cardiomyocytes that show a tendency to aggregate and form cell-cell contacts in preference to contact with soft gels (41). Such studies may set the stage for a better understanding of mechanosensitivity in cell-cell interactions during embryogenic and tissue regeneration processes.

Materials ranging from fibrin gels and microfabricated pillars to layer-by-layer polymer assemblies (41-45) all suggest a similar trend of more organized cytoskeleton and larger, more stable adhesions with increasing $E$ as outlined here, despite likely differences in adhesive ligand density and long-time elasticity. However, the responses appear to be specific to anchorage-dependent and/or relatively contractile cells. Highly motile amoeboid cells such as human neutrophils are perfectly viable in blood (a fluid) and do not appear to be sensitive to substrate stiffness; neutrophils spread on soft gels just as much as they do on stiff gels and glass, whether activated or not (46-48). Although additional study is needed and could prove ligand dependent, the initial contrast with cells derived from solid tissue highlights the compelling need for insights into molecular pathways of stiffness sensing in relation to anchorage dependence and contractility. Variation with cell type implies an active, regulated response, rather than a universal need of cells to exert traction forces on a stiff matrix. Differences no doubt depend in part on expression and engagement of adhesion molecules. Integrins reportedly undergo adhesion-modulating conformational changes in response to force (49), and they also appear to be down-regulated on soft gels [e.g., $\alpha_{5}$-integrin (40)]. However, overexpression of $\alpha_{5}$-integrin does not override the limited spreading of cells on soft gels, whereas overexpression of actin drives cytoskeletal assembly and strongly promotes spreading (17).

\section{Nonlinear Response to Compliance Signals and Molecular Effectors}

Myosin inhibitors-including a potent nonmuscle myosin II inhibitor, blebbistatin (50) - have provided key evidence for the critical role of contractility in substrate sensing $(14,38)$. Important roles are also reported for integrating activator proteins of the Ras superfamily, especially Rho subfamily members that are broadly known to regulate the cytoskeleton, cell growth, and transcription. In cells such as fibroblasts, it is well established that Rho-stimulated contractility drives stress fiber and focal adhesion formation and that up-regulation of $\alpha$ smooth muscle actin correlates with contractility on rigid substrates $(33,51)$. Rac1 is another Rho family protein that when activated in macrophages, promotes engulfment of antibody-bearing soft beads, which otherwise are not engulfed (48). RhoA, 
in contrast, has no observable effect in these measurements. Current views of signaling pathways, especially various physical signals (Fig. 3A), clearly implicate Rac in cell motility (versus contractility) - indeed, myosin inhibition activates Rac (52). The involvement of contractile-effector proteins in sensing implies that cell crawling is also likely to be sensitive to substrate stiffness (Fig. 3B), as demonstrated in studies of the "cell on gel" effect with epithelial cells (14), fibroblasts (20), and smooth muscle cells $(53,54)$. With the latter cell type, crawling speed appears maximal at an intermediate stiffness. The result is reminiscent of a bell-shaped curve of crawling speed versus the concentration of adhesive ligand (55), which has been mathematically modeled as a shift in the balance between ligand-mediated traction and ligandmediated anchorage (56). Additionally, smooth muscle cells on gels are slowed by inhibition of Rho kinase, suggesting that RhoA activity contributes to the tensions needed to detach any established adhesions at the rear of a motile cell (a process not needed in engulfment) (57). The dependence of cell crawling speed and direction on substrate stiffness, particularly gradients in stiffness, is now referred to as "durotaxis" (20).

Molecular mechanisms involved in cellular responses to matrix stiffness are still open to investigation, but it seems important to consider close relationships (or not) between "inside $\rightarrow$ outside $\rightarrow$ in" pathways and "outside $\rightarrow$ in" pathways (Fig. 3A). Adhesions on stiff materials are multifaceted mechanosensors [for a review, see, e.g., (5)], and, on the one hand, contractility does appear to regulate the formation and dynamics of adhesion structures (14). Indeed, myosin II has a well-established role on rigid substrates in adhesion and cytoskeletal organization (33), as well as spreading (58) and cell tension (13). On the other hand, applying external forces to cells (outside $\rightarrow$ in) leads to growth of focal adhesions on rigid materials, with or without myosin contractile forces (59). Nonetheless, inside $\rightarrow$ outside activity can trigger outside $\rightarrow$ in pathways such as the opening
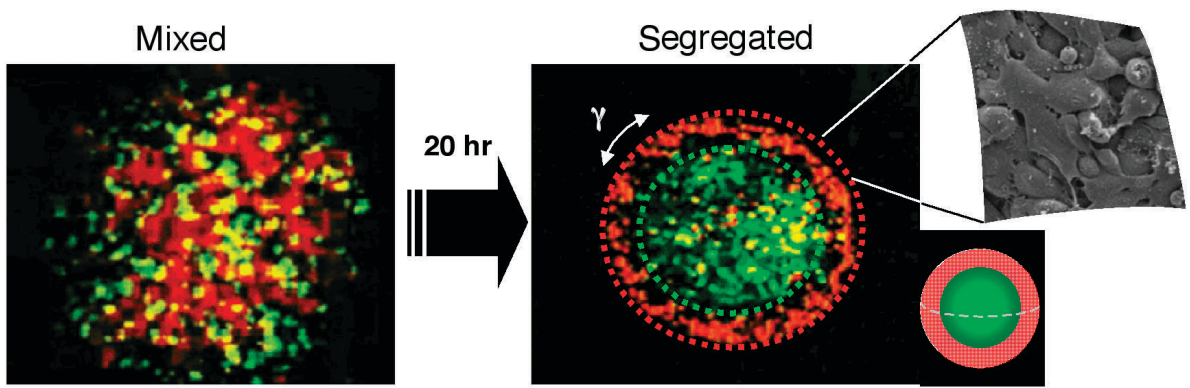

Fig. 4. Sorting of two cell types into a 3D shell-core aggregate $(\sim 300 \mu \mathrm{m}$ in diameter) in which low expressers of $\mathrm{N}$-cadherin (labeled in red) surround high expressers of $\mathrm{N}$-cadherin (labeled in green) (73). Scanning electron micrograph of a typical spheroid's surface shows well-spread cells. [Adapted from (73) with permission from Elsevier. Image courtesy of G. Forgacs, University of Missouri]

of stress-activated channels (60), with induction of calcium transients and activation of calmodulin and myosin II.

Additional work from the outside $\rightarrow$ in perspective has shown that stretching wellspread cells leads to deactivation of Rac (for $<30 \mathrm{~min}$ ) without affecting Rho activity (52). Stretching can also create new cytoskeletal binding sites for activator and adapter proteins (61) and thus alter the balance between protrusion and contractility. The mechanism may involve conformational changes to uncover scaffold binding sites or other activities; for example, one key focal adhesion protein, talin, must unfold for vinculin binding (62-64), and although the unfolding forces are not yet clear, similar helical bundle cytoskeletal proteins unfold at forces that just a few myosin molecules can generate. On the other hand, fluid shearing of endothelial cells activates Rho and also increases cell traction forces (65), but how such stimulationtransient or sustained - depends on myosin activity and compares with substrate-mediated pulling forces or substrate compliance effects remains unknown.

The effects and effectors of contractility can be transient as well as nonlinear, but are nonetheless essential to clarify. The temporary deactivation of Rac with stretch may have to be integrated over time to understand its place in signaling (66), and although myosin II activity is crucial for stiffness sensing, on rigid substrates it only delays the earliest phase of cell spreading (by $\sim 2$ hours), apparently through stiffening of the cell cortex (67). Overstimulation of myosin, like overstimulation of most motors, is also likely to slow and eventually stall cell migration. The effect may be related to the formation of less dynamic myosin assemblies on progressively stiffer substrates, fostering larger, more stable focal adhesions. Reconstitution experiments with mixtures of actin, myosin, adenosine $5^{\prime}$ triphosphate (ATP), and cross-linkers might lend important insight into motor-driven selfassembly processes.

Varied responses to mechanical signals at the cellular and molecular scales are increas-

18 NOVEMBER 2005 VOL 310 SCIENCE WWW.sciencemag.org ingly in need of multivariate analyses. More data are needed to define coupled responses to substrate stiffness, contractile state, ligand density, and activator levels, as well as effects such as growth factor stimulation. A number of studies have already revealed nonlinear response maps, as illustrated by the spread area of cells on gels (Fig. 3C). Modeling efforts to date have been thermodynamic $(17,68)$, kinetic (56), and-for cellcell interactions-purely mechanical (69), but all generally yield nontrivial responses, saturable or even bell-shaped in $E$ and other inputs. A major challenge in all such modeling is to clarify the principal enigma: how contractile traction forces exerted by a cell tend to increase with stiffness of the cell's substrate.

\section{Do Cells Feel Their Way in Organogenesis?}

Cell type-dependent increases in contractility with increasing substrate stiffness may offer partial answers to some long-standing questions of cell-cell organization. Random mixtures of two cell types often lead to shell-core cell aggregates (Fig. 4), as first observed when heart cells segregated into the interior of a mass of retinal cells after 1 day in culture $(70)$. Individual cell clusters form by "pulling" away from each other (71). Such observations are now being used to manipulate aggregate morphologies through printing of cell masses into gels as toroids and other shapes (72). Such phenomena have been explained by a "differential adhesion hypothesis" in which different cell types bear different numbers and types of adhesion proteins (e.g., cadherins), giving rise to an effective surface tension, $\gamma$, at interfaces with cell layers (73). Although possible contributions of cytoskeleton and cell tension have not yet been reported, studies of zebrafish embryos (74) have shown that (i) disruption of actin filaments dissociates cells entirely, even though cadherins remain at the cell surface; and (ii) the effect is potentiated by at least one drug that inhibits actomyosin contractility.

Quantitative estimates of $\gamma$ for the spherical aggregates of cadherin-expressing cells (73) exceed the rate-dependent cohesive strength of lipid bilayers [as low as 2 to $3 \mathrm{mN} / \mathrm{m}$ (75)] and suggest adhesion energies per cadherin that are orders of magnitude larger than would be expected of individual cadherin bonds. Such large $\gamma$ values could be due to the cytoskeleton or even contractility (because $\gamma /\langle\tau\rangle \cong 1$ to $10 \mu \mathrm{m}$ is a stress fiber length scale), especially because there is growing evidence of common RhoGTPase-cytoskeleton signaling among integrin- and cadherin-mediated adhesion (76-79). A major role for contractility in cell sorting was speculated long ago (80), but results reviewed here make it clear that contractile state can be strongly influenced by 
the stiffness of the anchoring substrate. Heart cells pulling on equally stiff heart cells can generate a positive and steady feedback on their cytoskeleton that may not occur when these cells pull on other tissue cell types. Cell aggregation of less differentiated cells such as some stem cells that assemble into "embryoid bodies" has yet to be studied with myosin inhibitors or related methods, but the principles may extend to stem cell differentiation, particularly because at least some stem cells express nonmuscle myosin II at levels similar to those of myoblasts (81).

\section{Added Facets and Prospects}

Mechanobiology is a broad field. Emphasized here is the recent recognition that most tissue cells not only adhere to but also pull on their microenvironment and thereby respond to its stiffness in ways that relate to tissue elasticity. Many emerging topics are not dealt with adequately in this brief review of substrate stiffness effects. These include in vitro models of fibrotic stiffening and related disease processes $(82,83)$; perturbed secretion and uptake $(84,85)$; $2 \mathrm{D}$ versus $3 \mathrm{D}$ responses $(32,86)$; deformations of fibronectin and other matrix molecules (87); structure formation such as capillary development $(15,88)$; deeper aspects of cell differentiation such as with stem cells (81); the relative sensitivity and contractility of some cells relative to others; and broader effects of matrix elasticity, as well as fluidity (i.e., matrix rheology), on cells in tissue development, remodeling, and regeneration. For the cell biologist, this review may suggest the need for a better understanding of mechanochemical pathways and the benefit of more biologically relevant elastic substrates than rigid coverslips and polystyrene for in vitro studies. For the applied biologist or bioengineer, modified strategies for tissue repair and cell scaffolding may emerge, such as the development of fibrous scaffolds for cell seeding (23), where careful attention can be given to fiber flexibility. All of these topics seem likely to add to our rapidly growing recognition that tissue cells feel and respond to the mechanics of their substrate in many contexts.

\section{References and Notes}

1. E. Ruoslahti, M. D. Pierschbacher, Science 238, 491 (1987).

2. H. L. Hadden, C. A. Henke, Am. J. Respir. Crit. Care Med. 162, 1553 (2000)

3. For example, classic palpation self-exam reveals that tumor stiffening can be quantitated by both MRI (4) and ultrasound elastography (5).

4. A. L. McKnight et al., AJR Am. J. Roentgenol. 178 , 1411 (2002).

5. J. Bercoff et al., Ultrasound Med. Biol. 29, 1387 (2003).

6. D. R. Welch et al., Cancer Res. 60, 1552 (2000).

7. A. K. Harris, P. Wild, D. Stopak, Science 208, 177 (1980).

8. Since (7), methods for measuring traction force have become more quantitative by embedding beads in either films (9) or gels (10) or by using deformable patterns (11) or posts (12).

9. T. Oliver, M. Dembo, K. Jacobson, J. Cell Biol. 145 589 (1999)

10. W. A. Marganski, M. Dembo, Y. Wang, Methods Enzymol. 361, 197 (2003).

11. N. Q. Balaban et al., Nat. Cell Biol. 3, 466 (2001).

12. J. L. Tan et al., Proc. Natl. Acad. Sci. U.S.A. 100, 1484 (2003).

13. F. J. Alenghat, D. E. Ingber, Sci. STKE 119, pe6 (2002).

14. (Fibroblasts and epithelial cells) R. J. Pelham, Y. Wang. Proc. Natl. Acad. Sci. U.S.A. 94, 13661 (1997) with Erratum 95, 12070a (1998).

15. (Endothelial cells) C. F. Deroanne, C. M. Lapiere, B. V. Nusgens., Cardiovasc. Res. 49, 647 (2001).

16. (Transformed cells) H.B. Wang, M. Dembo, Y. Wang., Am. J. Physiol. Cell Physiol. 279, C1345 (2000).

17. (Smooth muscle cells) A. Engler et al. Biophys. J. 86, 617 (2004).

18. R. E. Mahaffy, C. K. Shih, F. C. MacKintosh, J. Kas, Phys. Rev. Lett. 85, 880 (2000)

19. The Hertz model for a spherical probe of radius $R$ indenting a thick slab gives $E \sim f / d^{3 / 2} R^{1 / 2}$ where the force $f$ indents to a depth $d$. The shear modulus, $G$ of an isotropic, incompressible material is $G \approx E / 3$, reflecting the fact that shearing and tensile extension are related by a rotation of frame.

20. C. M. Lo, H. B. Wang, M. Dembo, Y. Wang, Biophys. J. 79, 144 (2000).

21. C. Storm, J. J. Pastore, F. C. MacKintosh, T. C. Lubensky, P. A. Janmey, Nature 435, 191 (2005).

22. Y. C. Fung, A First Course in Continuum Mechanics: For Physical and Biological Engineers and Scientists (Prentice Hall, Englewood Cliffs, NJ, ed. 3, 1994).

23. M. M. Stevens, J. George, Science 310, 1135 (2005).

24. A. Gefen, S. S. Margulies, J. Biomech. 37, 1339 (2004).

25. S. Hu et al., Am. J. Physiol. Cell Physiol. 287, C1184 (2004).

26. G. Bao, S. Suresh, Nat. Mater. 2, 715 (2003).

27. F. Wottawah et al., Phys. Rev. Lett. 94, 098103 (2005).

28. (Skeletal Muscle Cells) A. J. Engler et al., J. Cell Biol. 166, 877 (2004)

29. Y. Yoshikawa, T. Yasuike, A. Yagi, T. Yamada, Biochem. Biophys. Res. Commun. 256, 13 (1999).

30. S. Diridollou et al., Skin Res. Technol. 6, 214 (2000).

31. W. M. Leader, D. Stopak, A. K. Harris, J. Cell Sci. 64, 1 (1983).

32. E. Cukierman, R. Pankov, D. R. Stevens, K. M. Yamada Science 294, 1708 (2001).

33. M. Chrzanowska-Wodnicka, K. Burridge, J. Cell Biol. 133, 1403 (1996).

34. O. J. Pletjushkina et al., Cell Motil. Cytoskeleton 48 235 (2001).

35. N. Wang et al., Proc. Natl. Acad. Sci. U.S.A. 98, 7765 (2001).

36. P. C. Bridgman, S. Dave, C. F. Asnes, A. N. Tullio, R. S Adelstein, J. Neurosci. 21, 6159 (2001).

37. (Neurons) L. A. Flanagan, Y. E. Ju, B. Marg, M. Osterfield, P. A. Janmey, Neuroreport 13, 2411 (2002).

38. M. A. Griffin, S. Sen, H. L. Sweeney, D. E. Discher J. Cell Sci. 117, 5855 (2004)

39. P. C. Georges, P. A. Janmey, in preparation.

40. T. Yeung et al., Cell Motil. Cytoskeleton 60, 24 (2005).

41. Y. Wang, in preparation.

42. P. C. Georges, P. A. Janmey, J. Appl. Physiol. 98, 1547 (2005).

43. G. P. Raeber, M. P. Lutolf, J. A. Hubbell, Biophys. J. 89 1374 (2005).

44. A. Saez, A. Buguin, P. Silberzan, B. Ladoux, Biophys J., in press

45. A. Engler, L. Richert, J. Wong, C. Picart, D. E. Discher Surf. Sci. 570, 142 (2004).

46. Phagocytosis of rigid yeast particles by neutrophils involves a large contractile stress $(\sim 1 \mathrm{kPa})$ and implicates myosin (47), consistent with stiffness sensitivity of phagocytosis by macrophages (48)

47. E. A. Evans, A. Leung, D. Zhelev, J. Cell Biol. 122 1295 (1993)
48. (Macrophages) K. A. Beningo, Y. Wang, J. Cell Sci. 115, 849 (2002)

49. M. Jin, I. Andricioaei, T. A. Springer, Structure (Cambridge) 12, 2137 (2004).

50. A. F. Straight et al., Science 299, 1743 (2003).

51. B. Hinz, G. Celetta, J. J. Tomasek, G. Gabbiani, C Chaponnier, Mol. Biol. Cell 12, 2730 (2001).

52. A. Katsumi et al., J. Cell Biol. 158, 153 (2002).

53. (Smooth muscle cells) S. R. Peyton, A. J. Putnam, J. Cell. Physiol. 204, 198 (2005).

54. N. Zaari, P. Rajagopalan, S. K. Kim, A. J. Engler, J. Y. Wong, Adv. Mater. 16, 2133 (2004).

55. S. L. Goodman, G. Risse, K. von der Mark, J. Cell Biol. 109, 799 (1989).

56. M. H. Zaman, R. D. Kamm, P. Matsudaira, D. A. Lauffenburger, Biophys. J. 89, 1389 (2005).

57. P. Y. Jay, P. A. Pham, S. A. Wong, E. L. Elson, J. Cell Sci. 108, 387 (1995).

58. D. Riveline et al., J. Cell Biol. 153, 1175 (2001).

59. L. P. Cramer, T. J. Mitchison, J. Cell Biol. 131, 179 (1995).

60. A. Doyle, W. Marganski, J. Lee, J. Cell Sci. 117, 2203 (2004).

61. M. Tamada, M. P. Sheetz, Y. Sawada, Dev. Cell 7, 709 (2004).

62. I. Fillingham et al., Structure (Cambridge) 13, 65 (2005).

63. Recent unfolding of spectrin helical bundles at force levels that myosins can generate are described in (64).

64. R. Law et al., Biophys. J. 85, 3286 (2003)

65. Y. T. Shiu et al., Biophys. J. 86, 2558 (2004).

66. A. R. Asthagiri, C. A. Reinhart, A. F. Horwitz, D. A. Lauffenburger, J. Cell Sci. 113, 4499 (2000).

67. T. Wakatsuki, R. B. Wysolmerski, E. L. Elson, J. Cell Sci. 116, 1617 (2003).

68. A. Nicolas, B. Geiger, S. A. Safran, Proc. Natl. Acad. Sci. U.S.A. 101, 12520 (2004).

69. I. B. Bischofs, U. S. Schwarz, Proc. Natl. Acad. Sci. U.S.A. 100, 9274 (2003)

70. M. S. Steinberg, Science 137, 762 (1962).

71. J. P. Trinkaus, J. P. Lentz, Dev. Biol. 89, 115 (1964).

72. K. Jakab, A. Neagu, V. Mironov, R. R. Markwald, G. Forgacs, Proc. Natl. Acad. Sci. U.S.A. 101, 2864 (2004).

73. R. A. Foty, M. S. Steinberg, Dev. Biol. 278, 255 (2005)

74. S. E. Zalik, E. Lewandowski, Z. Kam, B. Geiger, Biochem. Cell Biol. 77, 527 (1999).

75. E. A. Evans, V. Heinrich, F. Ludwig, W. Rawicz, Biophys. J. 85, 2342 (2003).

76. N. K. Noren, W. T. Arthur, K. Burridge, J. Biol. Chem. 278, 13615 (2003).

77. W. T. Arthur, N. K. Noren, K. Burridge, Biol. Res. 35 239 (2002).

78. In vitro studies of cadherin-coated surfaces show no cytoskeleton assembly and no response to contractile inhibitors (79).

79. H. Delanoe-Ayari, R. Al Kurdi, M. Vallade, D. GulinoDebrac, D. Riveline, Proc. Natl. Acad. Sci. U.S.A. 101 2229 (2004).

80. A. K. Harris, J. Theor. Biol. 61, 267 (1976).

81. A. Engler, S. Sen, H. L. Sweeney, D. E. Discher, in preparation.

82. R. G. Wells, J. Clin. Gastroenterol. 39, S158 (2005).

83. M. A. Griffin et al., J. Cell Sci. 118, 1405 (2005).

84. E. J. Semler, P. A. Lancin, A. Dasgupta, P. V. Moghe, Biotechnol. Bioeng. 89, 296 (2005).

85. H. J. Kong et al., Nat. Mater. 4, 460 (2005).

86. K. A. Beningo, M. Dembo, Y. Wang, Proc. Natl. Acad. Sci. U.S.A. 101, 18024 (2004)

87. G. Baneyx, L. Baugh, V. Vogel, Proc. Natl. Acad. Sci. U.S.A. 99, 5139 (2002).

88. A. L. Sieminski, R. P. Hebbel, K. J. Gooch, Exp. Cell Res. 297, 574 (2004).

89. Y. Shikinami, M. Okuno, Biomaterials 24, 3161 (2003).

90. P. F. Gratzer, J. P. Santerre, J. M. Lee, Biomaterials. 25, 2081 (2004).

91. K. Rottner, A. Hall, J. V. Small, Curr. Biol. 9, 640 (1999).

92. Support was provided by NIH, NSF-PECASE (D.E.D.), and Penn's NSF-MRSEC.

10.1126/science. 1116995 